\title{
Prevalence of internet addiction and its association with stressful life events and psychological symptoms among adolescent internet users
}

Tang Jie ${ }^{\mathrm{a}, \mathrm{b},{ }^{*}}$, Yu Yizhen ${ }^{\mathrm{b}}$, Du Yukai ${ }^{\mathrm{b}}$, Ma Ying $^{\mathrm{c}}$, Zhang Dongying ${ }^{\mathrm{a}}$,Wang Jiajia ${ }^{\mathrm{a}}$

a,School of Public Health, Guangzhou Medical University, Guangzhou, Guangdong, China

b, Department of Child, Adolescence \& Woman Health Care, School of Public Health, Tongji

Medical College, Huazhong University of Science \& Technology, Wuhan, Hubei, China

', Women and Children's Medical Center, Guangzhou, Guangdong, China

*, Corresponding author at: School of Public Health, Guangzhou Medical University,

Guangzhou, Guangdong, China. Tel./fax: +86 2083691906.

E-mail addresses: gytanjie@163.com (J. Tang), yuyizhen650@163.com (Y. Yu), duyukai2000@163.com (Y.Du),258257876@qq.com (Y. Ma), 1483600306@qq.com (D. Zhang), tylertangjie@163.com (J. Wang).

\section{Highlights}

1. There was a high prevalence of IA among Chinese adolescents.

2. Some stressful life events may be risk factors of IA.

3. Coping style mediate the association between stressful life events and IA.

4. No interaction between stressful life events and psychological symptoms. 


\begin{abstract}
Internet addiction (IA) among adolescents is a serious public health problem around the world. However, there have been few studies that examine the association between IA and stressful life events and psychological symptoms among Chinese adolescent internet users. We examined the association between IA and stressful life events and psychological symptoms among a random sample of school students who were internet users $(\mathrm{N}=755)$ in Wuhan, China. Internet addiction, stressful life events, coping style and psychological symptoms were measured by self-rated scales. The prevalence rate of internet addiction was $6.0 \%$ among adolescent internet users. Logistic regression analyses indicated that stressors from interpersonal problem and school related problem and anxiety symptoms were significantly associated with IA after controlling for demographic characteristics. Analyses examining the coping style with the IA revealed that negative coping style may mediate the effects of stressful life events to increase the risk of IA. However, no significant interaction of stressful life events and psychological symptoms was found. These findings of the current study indicate a high prevalence of internet addiction among Chinese adolescent internet users and highlight the importance of stressors from interpersonal problem and school related problem as a risk factor for IA which mainly mediated through negative coping style.
\end{abstract}

\title{
Keywords
}

Internet addiction; Psychological symptoms; Stress life events; Coping style; Adolescents Chinese 
Introduction

During the past two decades, internet users have increased consider-ably. Internet brings lots of convenience to modern life, however, the growth in internet use has also make many persons depend too much on internet which may be termed as internet addiction (IA) (Ko, Yen, Yen, Chen, \& Chen, 2012). IA can be defined as an inability to control one's use of the internet and led to negative consequences in daily life. The terms 'compulsive internet use', 'pathological internet use', 'problematic internet use', etc., were usually considered as the synonyms of IA, although their views may be different (American Psychiatric Association, 2010).

Several studies have examined the prevalence of IA during the past few years. For example, a study conducted in the USA reported a prevalence of 1.0\% (Aboujaoude, Koran, Gamel,Large,\&Serpe,2006), a study conducted in Europe reported a prevalence of $9.0 \%$ (Villella et al., 2011), and studies conducted in Asia reported prevalence ranging from 2.0\% to $18.0 \%$ (Ko, Yen, Yen, Lin, \& Yang, 2007; Wang, Wang, \& Fu, 2008). Al-though data from those studies reported inconsistent occurrence rate of IA, it is no doubt that IA has become a serious public health problem around the world, especially in adolescents (Christakis, 2010). Previous studies suggested that IA increased the risk to suffer from a number of negative social and health consequences, such as poor academic perfor-mance, poor personality relationship, anxiety, depression (Jenaro, Flores, Gómez-Vela, GonzálezGil, \& Caballo, 2007; Yang \& Tung, 2007), and other behavioral problems (Sung, Lee, Noh, Park, \& Ahn, 2013). Therefore, it is urgent to protect adolescents from addiction to internet use.

Few studies have examined the association between stressful life events and IA among adolescents (Xie, Zhou, Xu, \& Peng, 2010; Yan, Li, \&Sui,2013). Young proposed a theory that IA may be a temporary phenomenon for some individual, and is actually a behavioral pattern which plays a role in some of the negative cognitions that compensate for failed areas of life (Davis, 2001). According to this, difficulties accumulated in real-life may contribute to IA because internet provides an escape for the individual from stressful life events. However, coping style may play a significant, mediating role in this association (Schroevers, Kraaij, \& Garnefski, 2007), and the interaction of stressful life event and the psychological symptoms of adolescents may enhance or weaken this association, and there was no evidence that supports this hypothesis. 
The purposes of the present study are: (1) To investigate the prevalence of IA. (2) To examine the association between IA, stressful life events, psychological symptoms and coping style. (3) To explore the interactions of stressful life events and psychological symptoms on IA.

\section{Methods}

Data resource

The data was from a study on sub-health and risk behavior among adolescents, which was conducted in Wuhan, China. A detailed sam-pling process, data collecting process and ethics statements were de-scribed elsewhere (Tang et al., 2010). The final data analyzed in the present study consisted of 755 students, 504 boys and 251 girls with a mean age of 14.0 years $($ Standard Deviation $=1.8)$.

Measures

Internet addiction. The Chinese version of Young's Internet Addiction Test was administrated to assess IA. It is a self-rated test that contains 20 items, and each item was scored on a scale of $1-5$. IA was assessed by summing the scores and scores $\geq 50$ were classified as IA (Young, 1995). Data from a reported study indicated adequate reliability of YIAT in languages other than English (Cronbach alpha $\geq 0.90)$ (Guan, Isa, Hashim, Pillai, \& Singh, 2012; Khazaal et al., 2008).

Stressful life events. The Adolescent Self-Rating Life Events Checklist was used to assess individuals' subjective suffering from stressful life events experienced during the past 6 months. It includes 82 life events that were categorized into four types: interpersonal problems (e.g. felt misunderstood by others, humiliated in public or experienced discrimination by others), school related problems (e.g. failed an exam, had a heavy workload), family related problems (e.g. poor economy, death of parent, or parents' divorce), and personal related problems (e.g. dissatisfy of appearance, accidental injuries). Higher total scores on the scale represent a greater number of stressful life events experienced in the past 6 months (Wang, Hao, Tao, Zhu, \& Zhang, 2011). In the present study, the Cronbach's alpha for each subscale was $0.948,0.921,0.943$, and 0.935 .

Coping styles. The Chinese Trait Coping Style Questionnaire was used to measure positive and negative coping styles (Jiang \& Zhu, 1999). Each subscale has 10 items, rated from 1 to 5 . Higher total scores of the subscales rep-resent greater intensity of related coping 
style. In the present study, Cronbach's alpha values for positive and negative coping style scales were 0.833 and 0.821 , respectively.

Depressive symptoms. Respondents completed the 20-item Centre for Epidemiological Studies Depression Scale, which has been used extensively in studies of adolescents (Chen, Dunne, \& Han, 2006). Items are rated from 0 to 3, with higher scores indicating greater severity of depressive symptoms. In the present survey, the scale had a very good internal consistency $(\mathrm{a}=0.85)$.

Anxiety symptoms. The revised Self-Rating Anxiety Scale consists of 20 items, and each item was rated on a 4-point scale from 1 ('rarely')to4('always'). The raw score needs to be converted into an 'Anxiety Index' score (Zung, 1971), and a higher 'Anxiety Index' score reflected more severe anxiety. The internal consistency estimate in this study was 0.756 .

Statistical analysis

Descriptive statistics (mean, standard deviation, and percentage) were calculated to reflect the background characteristics of the sample. Chi-square tests and t-tests were employed to compare the composition of demographic characteristics and the scores of stressful life event, cop-ing style, depression symptoms and anxiety symptoms between the internet addicts and non-internet addicts. Logistic regression was used to explore the associations of stressful life events, psychological symptoms (depression and anxiety) and coping style (positive and negative coping) in a multivariate context. Product terms representing the interaction between stressful life event and psychological symptoms were also included in subsequent models. Significance level was set at 0.05 , and all tests were two sided. Statistical analyses were conducted using Statistical Package for Social Sciences software (SPSS for Windows 16.0, SPSS Inc., Chicago, IL).

\section{Results}

Demographic characteristics

General characteristics of the participants were summarized in Table 1. The scores of YIAT were significantly higher in boys than in girls $(t=2.725, p=0.007)$. However, the scores of negative coping, positive coping, depression symptom and anxiety symptom were more likely lower in boys than in girls $(\mathrm{t}=4.020,4.571,3.927,5.076, \mathrm{p} b \mathrm{~b} .05)$. No gender significance on total stressful life events and its' four dimensions was found ( $\mathrm{p} \mathrm{N} \mathrm{0.05).}$

Prevalence of internet addiction 
There were $45(6.0 \%)$ respondents who were classified as internet ad-dicts. The prevalence rate was significantly higher in boys than in girls $\left(7.5 \%\right.$ vs. $2.8 \%, \chi^{2}=6.747, \mathrm{p} \mathrm{b}$ $0.05)$ and with significantly higher number of urban students than rural students $(9.4 \%$ vs. $2.8 \%, \chi^{2}=14.758, \mathrm{p}$ b 0.05$)$. However, there was no significance between different age groups.

Association of IA with stressful life events and psychological symptoms

The internet addicts scored higher on the stressful life events, depression symptom and anxiety symptom than the non-addicts, however, significances were only found on interpersonal problems, school related problems and anxiety symptom $(\mathrm{t}=2.252,2.022$, 3.216, p b 0.05). Logistic regression analysis (Table 2, Model I) showed that interpersonal problems, school related problems and anxiety symptom were significantly associated with IA after adjusting for demographic characteristics.

The effects of coping style and interaction of stressful life event and psychological symptom

The scores of positive and negative coping styles were higher in internet addicts than in non-addicts, however, significance was only found on negative coping style. Logistic regression showed that negative coping style was also associated with IA after controlling demographic characteristics (Table 2, Model II). Interestingly, the odds ratios for interpersonal problems and school related problems were not change. To test whether the association between stressful life event and IA differed among those who had and did not had high score on psychological symptom, the interaction of stressful life events and anxiety or depression symptom was added to the regression equation presented in Table 2 (Model III). The coefficients for both of the two interactions were zero, indicating that there were no significant interactions of stressful life event and psychological symptom when predicting IA.

\section{Discussion}

Prevalence rate of IA among Chinese adolescents

In the present study, the prevalence of IA among the participants was $6.0 \%$. This rate was lower than that reported among middle school students in Wuhan using the same YIATC measurement, but was close to those studies conducted in other cities of China (Lam, Peng, Mai, \& Jing, 2009; Wang et al., 2011; Wu et al., 2013). Variations in the prevalence of IA could be due to different methodologies, the taxonomy of IA or the time frame of the performed research (Durkee et al., 2012). Additionally, the present study also showed that the prevalence of IA varies with gender and residence, which may be attributed to the type of 
internet activity and the accessible of internet (Charlton \& Danforth, 2007; Li, Wang, \& Wang, 2009).

Stressful life events, psychological symptoms and IA

Adolescents experienced increasing stressors with growth and different types of life events that have different characteristics: interpersonal problems and school related problems are stressors characterized by higher frequency of occurrence; and family related problems and personal problems are stressors characterized by lower frequency of occurrence. The present study found that life stress from interpersonal and school correlated positively with IA, which was consistent with a previous study (Li et al., 2009; Velezmoro, Lacefield, \& Roberti, 2010), and Li and his colleagues posited that life events that occur in higher frequency rather than life events that occur in lower frequency are induced to IA (H. Li et al., 2009). Those results may be also supported by clinical studies demonstrating that most human emotional and behavioral disorders, such as substance abuse, are induced by chronically mild stress (Sareen et al., 2007).

A positive relationship between IA and anxiety has also been observed among the study population. However, relationship between IA and depression was not found under a multivariate context, and this was inconsistent with previous studies (Cunming, 2010; Kranzler \& Rosenthal, 2003). Association between IA and psychological symptoms is very complex. Kraut and colleagues proposed a "rich get richer" model in which the internet provides more benefits to those who are al-ready well-adjusted and in contrast, poorly adjusted adolescents with depression may experience more deleterious effects with heavy inter-net use and may be vulnerable to become addicted to internet (Kraut et al., 2002).

A previous study reported that the interaction of stressful life events and psychological symptoms may increase the risk of IA (Yan et al., 2013). However, in the present study, we did not find the interaction of stressful life events and psychological symptoms that increase sus-ceptibility to IA. Therefore, more studies are needed to understand the interactions of stressful life events and psychological symptoms on IA.

The effects of coping style on IA

Coping style helps individuals to deal with everyday life stressors on both an emotional and a cognitive level, and an avoidant coping style could mediate the relationship between stressful life events and IA (Li, Zhang, Li, Zhen, \& Wang, 2010). Our results indicated that negative coping style could increase the risk of IA. Notably, the odds ratio of stressful life events to IA from positive coping style was lower than that from negative 
coping style, which implied that stressful life events that increase the risk of IA were possibly mediated indirectly by negative coping style.

\section{Limitations}

Several limitations should be noted in the present study. Firstly, the cross-sectional survey design is not allowing a conclusion to be drawn from the risk factors to IA among child and adolescents, so the results should be interpreted carefully. Secondly, IA lacks definite diagnostic criteria and was not determined by certified clinicians internationally. Young's Internet Addiction Test reflects mainly the DSM-IV's criteria of addiction, and the results may differ if other criteria were used (e.g., the International Statistical Classification of Diseases).

\section{Conclusion}

In sum, this study indicates a high prevalence of internet addiction among Chinese adolescent internet users, advances our understanding of the association of IA with stressful life events and psychological symptoms and provides evidences for the prevention of IA.

\section{Role of funding source}

This study was supported by the National High-Tech Research and Development Program ("863" Program) (Grant No. 2006AA02Z427). Opinions reflect those of the authors and not necessarily those of the granting agencies.

\section{Contributors}

JT, DY and YY participated in the design of the study and performed the statistical analysis. JT and WJ drafted the manuscript and YM and ZD participated in data collection. YY participated in its design and coordination and helped to draft the manuscript. All authors read and approved the final manuscript.

\section{Conflict of interest}

The authors declare that they have no conflict interest.

\section{Acknowledgments}

The authors express their gratitude to all participating schools and the participants for their cooperation in data collection. The authors also express their gratitude to Profes-sor (tenured) Qingyi Wei, Department of Epidemiology, Division of Cancer Prevention and Population Sciences, The University of Texas M. D. Anderson Cancer Center, Houston, TX, for his efforts of editing and revision. 


\section{References}

Aboujaoude, E., Koran, L. M., Gamel, N., Large, M.D., \& Serpe, R. T. (2006). Potential markers for problematic internet use: a telephone survey of 2,513 adults. CNS spectrums, 11,750

American Psychiatric Association (2010). APA announces draft diagnostic criteria for DSM5: New proposed changes posted for leading manual of mental disorders.

Available at: http://www.dsm5.org/Newsroom/Documents/Forms/AllItems.aspx Charlton, J. P., \& Danforth, I. D. (2007). Distinguishing addiction and high engagement in the context of online game playing. Computers in Human Behavior, 23, 1531-1548.

Chen, J., Dunne, M. P., \& Han, P. (2006). Child sexual abuse in Henan province, China: Associations with sadness, suicidality, and risk behaviors among adolescent girls. Journal of Adolescent Health, 38,544-549.

Christakis, D. (2010). Internet addiction: A 21st century epidemic? BMC medicine, 8,61. Cunming, H. (2010). The relationship between college students' network behavior and depression and anxiety. Medicine and Society, 11, 035.

Davis, R. A. (2001). A cognitive-behavioral model of pathological Internet use. Computers in Human Behavior, 17,187-195.

Durkee, T., Kaess, M., Carli, V., Parzer, P., Wasserman, C., Floderus, B., et al. (2012).Prevalence of pathological internet use among adolescents in Europe: Demographic and social factors. Addiction, 107,2210-2222.

Guan, N. C., Isa, S. M., Hashim, A. H., Pillai, S. K., \& Singh, M. K. H. (2012). Validity of the Malay version of the internet addiction test: A study on a group of medical students in Malaysia. Asia-Pacific Journal of Public Health.

Jenaro, C., Flores, N., Gómez-Vela, M., González-Gil, F., \& Caballo, C. (2007). Problematic internet and cell-phone use: Psychological, behavioral, and health correlates. Addiction research \& theory, 15,309-320.

Jiang, Q., \& Zhu, Y. (1999). Further explorations for a coping style questionnaire. Chin J Behav Med Sci, 8,167-169. 
Khazaal, Y., Billieux, J., Thorens, G., Khan, R., Louati, Y., Scarlatti, E., et al. (2008). French validation of the internet addiction test. CyberPsychology \& Behavior, 11,703-706.

Ko, C. -H., Yen, J. -Y., Yen, C. -F., Chen, C. -S., \& Chen, C. -C. (2012). The association between Internet addiction and psychiatric disorder: A review of the literature. European Psychiatry, 27,1-8.

Ko, C. -H., Yen, J. -Y., Yen, C. -F., Lin, H. -C., \& Yang, M. -J. (2007). Factors predictive for incidence and remission of internet addiction in young adolescents: A prospective study. CyberPsychology \& Behavior, 10,545-551.

Kranzler, H. R., \& Rosenthal, R. N. (2003). Dual diagnosis: Alcoholism and co-morbid psychiatric disorders. The American journal on addictions, 12,s26-s40.

Kraut, R., Kiesler, S., Boneva, B., Cummings, J., Helgeson, V., \& Crawford, A. (2002). Internet paradox revisited. Journalof socialissues, 58,49-74.

Lam, L. T., Peng, Z. -w, Mai, J. -c, \& Jing, J. (2009). Factors associated with Internet addiction among adolescents. CyberPsychology \& Behavior, 12,551-555.

Li, H., Wang, J., \& Wang, L. (2009). A survey on the generalized problematic Internet use in Chinese college students and its relations to stressful life events and coping style. International Journal of Mental Health and Addiction, 7,333-346.

Li, D., Zhang, W., Li, X., Zhen, S., \& Wang, Y. (2010). Stressful life events and problematic Internet use by adolescent females and males: A mediated moderation model. Computers in Human Behavior, 26,1199-1207.

Sareen, J., Cox, B. J., Stein, M. B., Afifi, T. O., Fleet, C., \& Asmundson, G. J. (2007). Physical and mental comorbidity, disability, and suicidal behavior associated with posttraumatic stress disorder in a large community sample. Psychosomatic Medicine, 69, $242-248$.

Schroevers, M., Kraaij, V., \& Garnefski, N. (2007). Goal disturbance, cognitive coping strategies, and psychological adjustment to different types of stressful life event. Personality and Individual Differences, 43,413-423. 
Sung, J., Lee, J., Noh, H. -M., Park, Y. S., \& Ahn, E. J. (2013). Associations between the risk of internet addiction and problem behaviors among Korean adolescents. Korean journal of family medicine, 34,115-122.

Tang, J., Yu, Y., Du, Y., Ma, Y., Zhu, H., \& Liu, Z. (2010). Association between actual weight status, perceived weight and depressive, anxious symptoms in Chinese adolescents: A cross-sectional study. BMC public health, 10,594.

Velezmoro, R., Lacefield, K., \& Roberti, J. W. (2010). Perceived stress, sensation seeking, and college students' abuse of the Internet. Computers in Human Behavior, 26, 1526-1530.

Villella, C., Martinotti, G., Di Nicola, M., Cassano, M., La Torre, G., Gliubizzi, M.D., et al. (2011). Behavioural addictions in adolescents and young adults: Results from a prev-alence study. Journal of Gambling Studies, 27,203-214.

Wang, H., Hao, J., Tao, F., Zhu, P., \& Zhang, J. (2011). Mediator effects of negative coping styles between stressful life events and sub-health symptoms. Chinese Journal of School Health, 32,943-945.

Wang, Y., Wang, J., \& Fu, D. (2008). Epidemiological investigation on Internet addiction among Internet users in elementary and middle school students. Chinese Mental Health J, $22,678-682$.

Wang, H., Zhou, X., Lu, C., Wu, J., Deng, X., \& Hong, L. (2011). Problematic internet use in high school students in Guangdong province, China. PloS one, 6,e19660.

Wu, X., Chen, X., Han, J., Meng, H., Luo, J., Nydegger, L., et al. (2013). Prevalence and factors of addictive Internet use among adolescents in Wuhan, China: Interactions of parental relationship with age and hyperactivity-impulsivity. PloS one, 8,e61782.

Xie, Y., Zhou, P., Xu, L., \& Peng, Z. (2010). Prevalence of internet addiction and the related factors in middle school students in Guangzhou. Journal of Southern Medical University, $30,1801-1804$.

Yan, W., Li, Y., \& Sui, N. (2013 Apr 25). The relationship between recent stressful life events, personality traits, perceived family functioning and internet addiction among college students. Stress and Health, http://dx.doi.org/10.1002/smi.2490. 
Yang, S.C., \& Tung, C. -J. (2007). Comparison of Internet addicts and non-addicts in

Taiwanese high school. Computers in Human Behavior, 23,79-96.

Young, S. -K. (1995). Internet addiction test. Available at: http://netaddiction.com/Zung, W.

W. (1971). A rating instrument for anxiety disorders. Psychosomatics, 12, 371-379.

Table 1 Demographic characteristics and risk factors of the study sample

\begin{tabular}{|c|c|c|c|}
\hline & Boys $(n=504)$ & Girls $(n-=251)$ & All $(n=755)$ \\
\hline \multicolumn{4}{|l|}{ Age group (n, \%) } \\
\hline 10-12 years & $127(25.2)$ & $78(31.1)$ & $205(27.2)$ \\
\hline 13-15 years & $252(50.0)$ & $123(49.0)$ & $375(49.7)$ \\
\hline 16-19 years & $125(24.8)$ & $50(19.9)$ & $175(23.2)$ \\
\hline \multicolumn{4}{|l|}{ Residence (n, \%) } \\
\hline Urban & $234(46.4)$ & $126(50.2)$ & $360(47.7)$ \\
\hline Rural & $269(53.4)$ & $125(49.8)$ & $394(52.2)$ \\
\hline YIAT-C scores $(\mathrm{M} \pm \mathrm{SD})^{\mathrm{a}}$ & $30.5 \pm 12.4$ & $28.2 \pm 10.2$ & $29.7 \pm 11.8$ \\
\hline Total stressful life events $(\mathrm{M} \pm \mathrm{SD})$ & $147.3 \pm 94.5$ & $150.1 \pm 87.9$ & $148.2 \pm 92.3$ \\
\hline Interpersonal problem $(\mathrm{M} \pm \mathrm{SD})$ & $26.7 \pm 23.8$ & $26.2 \pm 23.5$ & $26.6 \pm 23.7$ \\
\hline School related problem $(\mathrm{M} \pm \mathrm{SD})$ & $51.8 \pm 28.5$ & $53.1 \pm 25.0$ & $52.2 \pm 27.4$ \\
\hline Family Related problem $(\mathrm{M} \pm \mathrm{SD})$ & $43.0 \pm 31.1$ & $45.5 \pm 30.8$ & $43.8 \pm 31.0$ \\
\hline Personal problem $(\mathrm{M} \pm \mathrm{SD})$ & $25.8 \pm 21.3$ & $25.2 \pm 21.5$ & $25.6 \pm 21.4$ \\
\hline \multicolumn{4}{|l|}{ Copying style } \\
\hline Negative coping $(\mathrm{M} \pm \mathrm{SD})^{\mathrm{a}}$ & $20.7 \pm 7.0$ & $23.3 \pm 7.8$ & $21.6 \pm 7.4$ \\
\hline Positive coping $(\mathrm{M} \pm \mathrm{SD})^{\mathrm{a}}$ & $26.5 \pm 7.5$ & $28.9 \pm 7.5$ & $27.3 \pm 7.6$ \\
\hline Depression symptom $(\mathrm{M} \pm \mathrm{SD})^{\mathrm{a}}$ & $37.2 \pm 8.4$ & $39.4 \pm 7.2$ & $37.9 \pm 8.1$ \\
\hline Anxiety symptom $(\mathrm{M} \pm \mathrm{SD})^{\mathrm{a}}$ & $31.3 \pm 7.0$ & $34.0 \pm 6.6$ & $32.2 \pm 7.0$ \\
\hline
\end{tabular}

Note: YIAT-C: Young's Internet Addiction Test, Chinese version. a, $p<0.05$

Table 2 Logistic regression models predicting internet addiction

\begin{tabular}{lcccccc}
\hline & \multicolumn{2}{c}{ Model 1 } & \multicolumn{2}{c}{ Model 2 } & \multicolumn{2}{c}{ Model 3 } \\
\cline { 2 - 7 } & OR & $95 \%$ CI & OR & 95\%CI & OR & $95 \%$ CI \\
\hline Age in years & 0.94 & $(0.78-1.14)$ & 0.97 & $(0.97-1.18)$ & 0.96 & $(0.78-1.18)$ \\
Gender (female vs. male) & 0.28 & $(0.12-0.65)^{\mathrm{a}}$ & 0.17 & $(0.07-0.44)^{\mathrm{a}}$ & 0.17 & $(0.07-0.44)^{\mathrm{a}}$ \\
Residence (urban vs. rural) & 4.57 & $(2.17-9.64)^{\mathrm{a}}$ & 5.35 & $(2.44-11.71)^{\mathrm{a}}$ & 5.35 & $(2.44-11.71)^{\mathrm{a}}$ \\
Depressive symptoms scores & 1.01 & $(0.95-1.05)$ & 0.96 & $(0.91-1.02)$ & 0.90 & $(0.81-1.01)$ \\
Anxiety symptoms score & 1.06 & $(1.02-1.11)^{\mathrm{a}}$ & 1.03 & $(0.97-1.09)$ & 1.00 & $(0.89-1.11)$ \\
Interpersonal problem & 1.04 & $(1.02-1.07)^{\mathrm{a}}$ & 1.04 & $(1.02-1.07)^{\mathrm{a}}$ & 1.05 & $(1.02-1.07)^{\mathrm{a}}$ \\
\hline
\end{tabular}




\begin{tabular}{ccccccc}
\hline School related problem & 1.03 & $(1.01-1.06)^{\mathrm{a}}$ & 1.03 & $(1.02-1.06)^{\mathrm{a}}$ & 1.05 & $(1.02-1.08)^{\mathrm{a}}$ \\
Family Related problem & 1.01 & $(0.99-1.02)$ & 1.00 & $(0.98-1.02)$ & 0.98 & $(0.95-1.01)$ \\
Personal problem & 0.99 & $(0.96-1.01)$ & 0.98 & $(0.96-1.00)$ & 0.97 & $(0.95-1.01)$ \\
Negative coping & -- & -- & 1.15 & $(1.09-1.20)^{\mathrm{a}}$ & 1.19 & $(1.12-1.26)^{\mathrm{a}}$ \\
Positive coping & -- & -- & 0.97 & $(0.92-1.03)$ & 0.99 & $(0.94-1.05)$ \\
Stressful life event $*$ depression & -- & -- & -- & & 1.00 & $(0.99-1.01)$ \\
Stressful life event $*$ anxiety & -- & -- & -- & & 1.01 & $(0.98-1.04)$ \\
Constant & -5.49 & & -6.38 & & -7.95 & \\
\hline
\end{tabular}

